\title{
Plasma emission correction in reflectivity spectroscopy during sputtering deposition
}

\author{
Iryna Gozhyk ${ }^{1}$, Letian Dai ${ }^{1}$, Quentin Hérault ${ }^{1}$, Rémi \\ Lazzari $^{2}$, Sergey Grachev ${ }^{1}$ \\ ${ }^{1}$ Surface du Verre et Interfaces, UMR 125 CNRS/Saint-Gobain Recherche, 39 \\ quai Lucien Lefranc, 93303 Aubervilliers, France \\ ${ }^{2}$ CNRS UMR 7588, Sorbonne Université, Institut des NanoSciences de Paris, 4 \\ place Jussieu, 75005 Paris, France \\ E-mail: iryna.gozhyk@saint-gobain.com
}

\begin{abstract}
Surface differential reflectivity spectroscopy is a fast non-destructive in situ and real-time measurement technique which allows following the first stages of thin film deposition. However, when applied to sputtering technique, spectra can strongly be distorted by residual light coming from plasma in a way, as shown herein, that depends on sample reflectivity. Thanks to suitable measurements, before and after growth with and without plasma or illumination lights, a protocol of signal correction is proposed to get rid of the spurious plasma contribution. The interest of the method is illustrated in the case of silver deposition on a silicon substrate.
\end{abstract}

Keywords Surface Differential Reflectivity Spectroscopy, sputtering deposition, UVvisible

Submitted to: J. Phys. D: Appl. Phys. 


\section{Introduction}

Sputtering deposition is the technique of choice of the coating industry due to its versatility and its scalable character. It requires the generation of a plasma, in general of a noble gas (but not only as in the case of reactive sputtering), to sputteroff material to be deposited from a target. This technique differs from evaporation by the presence of energetic species with a wide angular spread, the ion bombardment of the substrate and a quite fast growth rate (up to several tens of $\mathrm{nm} \cdot \mathrm{s}^{-1}$ ). Vast range of inorganic materials (including metals and dielectrics) can be deposited by magnetron sputtering on any kind of substrates, crystalline or amorphous such as glass panes. Various deposition parameters allow for an accurate control of the thickness and the microstructure of the growing polycristalline films: gas type and pressure, power applied to the target, presence of an external magnetic field in the case of magnetron sputtering, nature of the substrate, bias applied to the sample, sample-target distance, pulsing of the power, etc. But the harsh environment due to the plasma and the fast growth rate limits significantly the number of in situ and real-time monitoring tools applicable to sputtering. Among them, measurements of resistivity (to determine the percolation threshold in conductive films [1]) or stress [2,3] are compatible with sputtering deposition technique. Although ex situ film characterizations are always possible, they can only provide a limited amount of data points and may be hampered by the reactivity of the layers in the presence of atmosphere. They can also miss postgrowth relaxation effects $[2,3]$.

In fact, optics [4] offers an elegant way to tackle this question of monitoring, in particular during the earliest stages of growth before percolation. This is especially relevant since, to some extend, the final grain microstructure of the film and therefore its electrical, mechanical or optical properties are driven by the initial nucleation and growth of isolated islands in the case of Volmer-Weber growth. Moreover, some of the in situ measurement techniques such as resistivity are not sensitive to the beginning of growth. Spectroscopic ellipsometry $[5,6,7,8,9,10,11,12]$, surface differential reflectivity spectroscopy (SDRS) in the UV-visible [13, $14,15,16,17,2]$ or infrared ranges [18] or laser interferometry [19] have already been successfully employed during the course of sputtering deposition.
The principle of SDRS, as used herein, is to monitor in situ and in real-time changes in the reflectivity of a sample during modifications of its surface. Typical examples of the applications of this optical spectroscopy are gas adsorption and layer growth, the latter being the topic of the present work. The sensitivity of the technique lies in the dielectric contrast between the deposited material and the substrate, in particular when performed close to the Brewster's angle in p-polarization. To first order, the SDRS signal is directly proportional to the imaginary part of the dielectric function of the growing film [20] or more precisely to a combination of the polarizabilities and the morphology of growing entities [21, 22, 23]. At the origin, SDRS was devised to measure the difference of reflectivity between two parts of a sample via an oscillating sample/beam $[24,25]$. Then SDRS technique was extended to modulation spectroscopy [26] (for example, thermo, piezo or electro-modulations), where the applied external field was used to probe the electronic properties of semiconductors or metallic alloys. Sufficiently stable optical benches and excitation sources led to the application of SDRS to the study of gas adsorption or layer growth in vacuum chambers [27]. Finally, the advent of low-cost multichannel spectrometers allowed for drift correction from source [28] and polarization dependent measurements $[28,29]$. For a long time, the SDRS studies of thin film growth were mainly restricted to vapor deposition. But the fast detector readout paved the way for online monitoring of fast processes such as sputtering [17] at a time scale of the millisecond.

SDRS offers the possibility to monitor the excitonic absorptions $[30,31]$ of molecular assemblies on surfaces or plasmonic resonances of supported metallic nanoparticles [32, 33, 34, 35]. Associated with suitable dielectric modeling $[22,36,37,38,23]$, it was shown to be an invaluable tool to discuss quantitatively the processes of nucleation, growth and coalescence of metallic nanoparticles [32, 33, 34, 17, 39, 35] through the extreme sensitivity of the plasmonic absorptions to morphology.

In vapor deposition, the light coming from the crucible heater can be effectively shielded. But in the case of sputtering deposition, plasma emission is unavoidable and can strongly perturb or even distort the optical measurements. Up to now two solutions were used to overcome this issue: a spatial filter with a pinhole to look only at reflected light [17] or a high power 
probe source (such as Xe lamp [13, 15, 16, 2]). These solutions come at the expense of either overall intensity or stability in the UV range. Based on the hypothesis of a stable plasma, the goal of this work is to devise a robust strategy of measurements and correction of the SDRS signal from plasma emission. The interest of the method is tested on the island growth of silver on a silicon substrate.

\section{Experimental}

Experiments have been performed in a magnetron sputtering set-up encompassing three connected vessels: a load-lock, a photoemission chamber and a deposition chamber (base vacuum of $10^{-7}$ mbar) equipped with three cylindral magnetron targets (size 2 inches). Silver has been deposited in direct current mode at various powers $(50-200 \mathrm{~W})$ from a $99.999 \%$ pure target in Ar gas (purity $99.999 \%$ ) at a pressure of $1.710^{-3} \mathrm{mbar}$ and at a sample-target distance of $150 \mathrm{~mm}$. Presputtering of the target was systematically performed to clean it. Deposition was controlled by a fast shutter in front of the target. While the back side of the $\mathrm{Si}(100)$ substrate (size 2 inches, thickness $\sim 0.3 \mathrm{~mm}$, resistivity $1-20 \Omega . \mathrm{cm}$ ) was tarnished to avoid multiple reflections, the polished front side was covered by its native $\mathrm{SiO}_{2}$ oxide the thickness of which was estimated to $2 \mathrm{~nm}$ by ellipsometry (on a J.A Woolam ESM-300 apparatus). Substrates were used as received just after dust blowing with dry nitrogen. Silver deposition rate was calibrated from the thickness of the silver film measured by tapping atomic force microscopy (Bruker Icon AFM Scanner) on a trench obtained by dissolving a felt-tip pen stroke in acetone. Accounting for slight spatial inhomogeneities of thickness, it amounted to $0.48 \pm 0.01$, $0.84 \pm 0.01,1.27 \pm 0.02$ and $1.64 \pm 0.02 \mathrm{~nm} . \mathrm{s}^{-1}$ for powers of 50,100, 150 and $200 \mathrm{~W}$, respectively. On a test case of deposition at $50 \mathrm{~W}$ during $30 \mathrm{~s}$, electron probe micro-analysis, ellipsometry and AFM gave the expected value of $14.5 \mathrm{~nm}$ within $1 \mathrm{~nm}$.

The used SDRS set-up which is sketched in figure 1 is identical to that described in depth in reference [28]. Briefly, the light emitted by a deuterium-halogen lamp is focused on the sample through a set of lenses and a UV-transparent silica viewport mounted on the sputtering chamber. About one inch of the substrate is illuminated and all this area is imaged onto the detection arm. In the chamber, the deposition flux is highly directional towards the substrate leading to an extremely small spurious deposition on the optical viewports. A part of the lamp signal derived in a Yshape optical fiber is used to correct for source drift. The incident angle is fixed at $45^{\circ}$ through chamber flanges. The reflected light is condensed and split by a barium-borate Wollaston polarizer into two beams corresponding to the two polarization states $\mathrm{s}$ and $\mathrm{p}$, which are then completely collected by focusing lenses into separate optical fibers. Each signal is analyzed by a multichannel wide band grating spectrometer equipped with $\mathrm{Si}$ charge coupled device detectors (from Avantes). Depending on settings, the useful spectral range extends from 1.5 to $4.5 \mathrm{eV}$ i.e $275 \mathrm{~nm}$ to $830 \mathrm{~nm}$. Due to the fast deposition speed, the sampling rate is set at $20 \mathrm{~Hz}$, each spectrum being the average of 25 acquisitions to improve signal/noise ratio. Therefore, in the present experiments, the thickness resolution ranged from $0.023 \mathrm{~nm}$ per spectrum at $0.48 \mathrm{~nm} . \mathrm{s}^{-1}$ deposition rate to $0.083 \mathrm{~nm}$ per spectrum at $1.64 \mathrm{~nm} \cdot \mathrm{s}^{-1}$ deposition rate. In contrast to our previous work [17], which used a space filter to collect only the light coming from the specular reflection from the sample, the present SDRS set-up is quite sensitive to spurious light coming from the sputtering plasma.

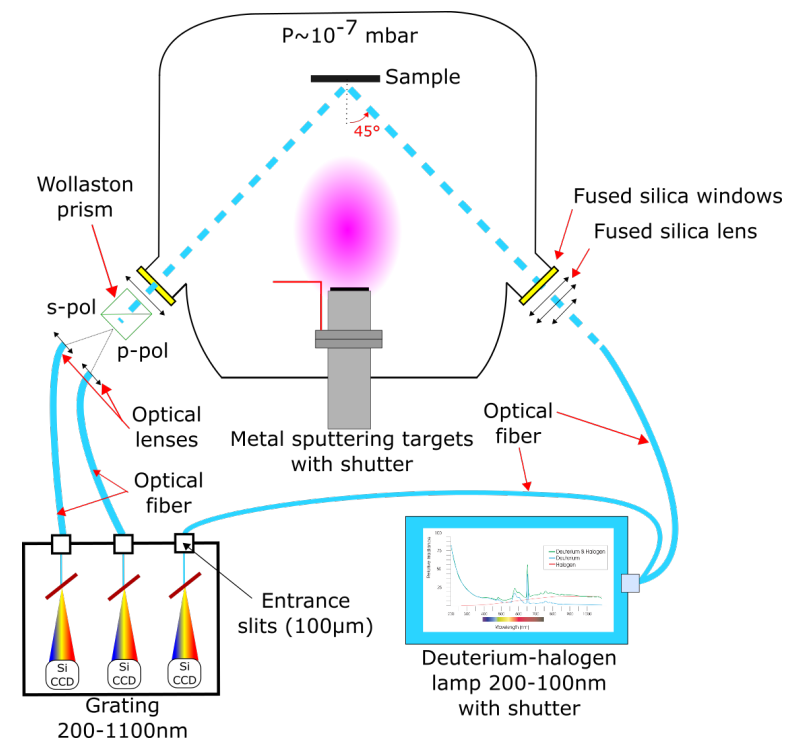

Figure 1. Schematic drawing of the set-up of surface differential reflectivity spectroscopy coupled to the sputtering deposition chamber. Most of the beam paths are guided into solarized optical fibres (blue lines).

\section{Sources of drift of differential reflectivity signal}

In SDRS, the quantity of interest is the relative variation of the sample reflectivity $\mathcal{R}$ as function of time or average film thickness $t$ (assuming constant deposition rate and unitary sticking coefficient):

$\frac{\Delta \mathcal{R}}{\mathcal{R}}(t)=\frac{\mathcal{R}(t)-\mathcal{R}_{s}}{\mathcal{R}_{s}}$,

where $\mathcal{R}_{s}=\mathcal{R}\left(t_{\text {ref }}\right)$ is the reflectivity of the native substrate before deposition the time of which is 
referred to as $t_{r e f}$. In the above equation and hereafter, the dependence of all quantities on the wavelength $\lambda$ (or the photon energy $E=h c / \lambda$ with $h$ the Planck constant and $c$ the speed of light) is implicit. To avoid the cumbersome determination of the absolute reflectivity, the actual measured quantity is the variation of the intensity $I(t)$ of the probe light reflected by the sample during deposition (hereafter referred to as time range $\left.\left[t_{\text {start }} ; t_{\text {stop }}\right]\right)$. In fact, $I(t)$ includes two terms:

$I(t)=\mathcal{R}(t) I_{\text {bench }}(t)+I_{\text {dark }}(t)$.

$I_{\text {bench }}(t)$ encompasses not only the spectral distribution of the probe light, but also the spectral imprint of all the elements of the optical bench (lenses, polarizers, windows of the sputtering chamber, etc...) including the spectrometer and its detector. $I_{\text {dark }}(t)$ is the so-called dark signal that is not related to the illumination by the probe light. Fortunately, for a optical set-up stable enough over time, the response of the optical bench can be assumed to be constant i.e. $I_{\text {bench }}(t) \simeq I_{\text {bench }}$. If the dark signal $I_{\text {dark }}(t)$ is also poorly time-dependent (i.e. $I_{\text {dark }}(t) \simeq I_{\text {dark }}$ ), the differential reflectivity is simply given by the relative variation of the dark-corrected signal $I_{c}(t)=I(t)-I_{\text {dark }}$ :

$\frac{\Delta \mathcal{R}}{\mathcal{R}}(t) \simeq \frac{I(t)-I\left(t_{\text {ref }}\right)}{I\left(t_{\text {ref }}\right)-I_{\text {dark }}}=\frac{\Delta I_{c}}{I_{c}}(t)$.

The normalization is done on the so-called reference signal $I_{c}\left(t_{r e f}\right)=I\left(t_{r e f}\right)-I_{\text {dark }}$ evaluated just before the start of film growth at $t=t_{\text {ref }}$. Still, in principle the signal drift over time is possible both through the response of the optical bench $I_{\text {bench }}(t)$ and through that of the dark signal $I_{\text {dark }}(t)$ (see equation 2). Their corrections or compensations require complex dedicated experimental set-ups. Double beam experiments have already been developed to correct for the bench drift. One part of the sample was hidden from deposition but still illuminated by the external source [40]. In such case, under the assumption of a stable dark, the signal measured on each of the two paths reads:

$I_{c}^{1}(t)=\mathcal{R}(t) I_{\text {bench }}^{1}(t)$

$I_{c}^{2}(t)=\mathcal{R}\left(t_{\text {ref }}\right) I_{\text {bench }}^{2}(t)$,

where $I_{\text {bench }}^{2}(t)$ is the reference signal from the area free of deposition. Under the hypothesis of similar drifts over the two beams:

$$
\begin{aligned}
\frac{\Delta \mathcal{R}}{\mathcal{R}}(t) & =\frac{I_{c}^{1}(t)}{I_{\text {bench }}^{1}(t)} \frac{I_{\text {bench }}^{1}\left(t_{r e f}\right)}{I_{c}^{1}\left(t_{r e f}\right)}-1 \\
& \simeq \frac{I_{c}^{1}(t)}{I_{c}^{1}\left(t_{r e f}\right)} \frac{I_{\text {bench }}^{2}\left(t_{r e f}\right)}{I_{\text {bench }}^{2}(t)}-1 \\
& \simeq \frac{I_{c}^{1}(t)}{I_{c}^{1}\left(t_{\text {ref }}\right)} \frac{I_{c}^{2}\left(t_{\text {ref }}\right)}{I_{c}^{2}(t)}-1 .
\end{aligned}
$$

Although the double beam experiments can be used to cancel also the temporal fluctuations of the signal, it is difficult to enforce on an experimental point of view as it requires (i) a beam splitter and a sample mask that may impact the deposition itself and (ii) similar beam drifts along the two paths. In the same spirit, if the thermal drift from the probe light source becomes the main source of bench drift, it can be corrected by simply monitoring the intensity of the probe light source over time as done in reference [28]. However, even for a stable optical bench over the deposition duration, drift from dark signal can be also an issue as in the case of sputtering. In the case of evaporation, the drift of the dark signal is nearly absent upon the opening of the cell shutter due to the generally good shielding of residual light coming from the heated crucible. But in the case of sputtering deposition, spurious light coming from the optical emission of the plasma contributing to $I_{\text {dark }}(t)$ can have dramatic effect. Several strategies can be developed to overcome this issue. The first one is to use a very intense source of light $[13,15,16,2]$ or a space filter [17] to look only at the reflected specular beam so that $I_{\text {dark }}(t) \ll \mathcal{R}(t) I_{\text {bench }}(t)$. Another possibility, which has not yet been implemented to the best of our knowledge, is to measure the reflected intensity and the dark signal in a stroboscopic way with respect to the probe light and at a time scale smaller than the drift of the plasma light. The subtraction of the two signals recorded with and without the probe light will give $I(t)-I_{\text {dark }}(t)$. Experimentally this could be achieved by a synchronization of the data acquisition on a fast shutter or a chopper that matches the sizable huge deposition rate. Beyond the implementation of the set-up, the main drawback of such an approach is the decrease of the final number of available spectra due to the loss of time for plasma recording and for synchronization; since sampling frequency is limited by signal/noise ratio, this could be an issue when one is interested in the first stages of film growth. The herein proposed strategy is different and is based on data processing assuming a stable plasma over the time scale of the deposit.

\section{Protocol of correction from plasma emission during sputtering deposition}

The proposed correction from plasma emission is based on a specific row of measurements and numerical treatments that are summarized in table 1 and described in what follows. The timeline of events with periods when the plasma or the probed light are present is given figure 2 .

In the case of sputtering deposition, manipulating 


\begin{tabular}{|c|c|c|c|c|c|c|}
\hline Stage of experiment & Time & Probe light & Plasma & Shutter & Type of measurement & Data obtained \\
\hline \multirow{4}{*}{$\begin{array}{c}\text { Before } \\
\text { deposition }\end{array}$} & $t<t_{\text {dark }}$ & $\mathrm{ON}$ & OFF & CLOSED & Optical alignment & No data \\
\hline & $t=t_{\text {dark }}$ & OFF & OFF & CLOSED & Actual dark signal & $I\left(t_{d a r k}\right)$ \\
\hline & $t=t_{\text {pre-sput }}$ & $\mathrm{OFF}$ & $\mathrm{ON}$ & CLOSED & $\begin{array}{c}\text { Plasma signal with closed shutter } \\
\text { to adapt dark signal in accordance } \\
\text { to measurement regime } \\
\text { (during or after deposition) } I\left(t_{\text {pre-sput }}\right)\end{array}$ & $I\left(t_{\text {pre-sput }}\right)$ \\
\hline & $t=t_{\text {ref }}$ & $\mathrm{ON}$ & $\mathrm{ON}$ & CLOSED & SDRS reference signal $I\left(t_{r e f}\right)$ & $I\left(t_{\text {ref }}\right)$ \\
\hline $\begin{array}{c}\text { During } \\
\text { deposition }\end{array}$ & $t_{\text {start }} \leq t \leq t_{\text {stop }}$ & $\mathrm{ON}$ & $\mathrm{ON}$ & OPEN & Signal $I(t)$ & $\left.\frac{\Delta I_{c}}{I_{c}}(t)=\frac{\left[I(t)-I\left(t_{\text {dark }}\right)\right]-\left[I\left(t_{\text {ref }}\right)-I\left(t_{\text {pre-sput }}\right)\right]}{I\left(t_{\text {ref }}\right)-I\left(t_{\text {pre }}\right)-s p u t}\right)$ \\
\hline $\begin{array}{l}\text { Suspended } \\
\text { deposition }\end{array}$ & $t=t_{\text {probe }}$ & $\mathrm{ON}$ & $\mathrm{ON}$ & CLOSED & Signal $I\left(t_{\text {probe }}\right)$ & 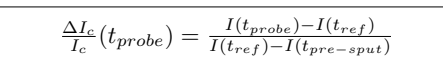 \\
\hline \multirow[t]{2}{*}{ Deposition } & $t=t_{p l}^{t}$ & $\mathrm{OFF}$ & $\mathrm{ON}$ & OPEN & $\begin{array}{c}\text { Plasma intensity in a regime } \\
\text { of thick film with nearly } \\
\text { constant reflectivity } I_{p l}^{t}\end{array}$ & 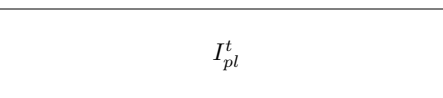 \\
\hline & $t=t_{p l}^{d}$ & $\mathrm{OFF}$ & $\mathrm{ON}$ & OPEN & $\begin{array}{l}\text { Direct contribution of plasma } \\
\text { radiation } I_{p l}^{d} \text { obtained } \\
\text { by moving the reflected beam } \\
\text { from collection direction }\end{array}$ & $I_{p l}^{d}$ \\
\hline Analysis & \multicolumn{5}{|c|}{ equation $11 \Longrightarrow \alpha$, equation $15 \Longrightarrow \beta$} & $\begin{array}{l}\text { Sample differential reflectivity } \\
\qquad \frac{\Delta R}{R}(t)=\frac{1}{\alpha}\left[\frac{\Delta I_{c}}{I_{c}}(t)-\beta\right]\end{array}$ \\
\hline
\end{tabular}

Table 1. he followed flow chart of measurements and treatments for plasma emission correction.

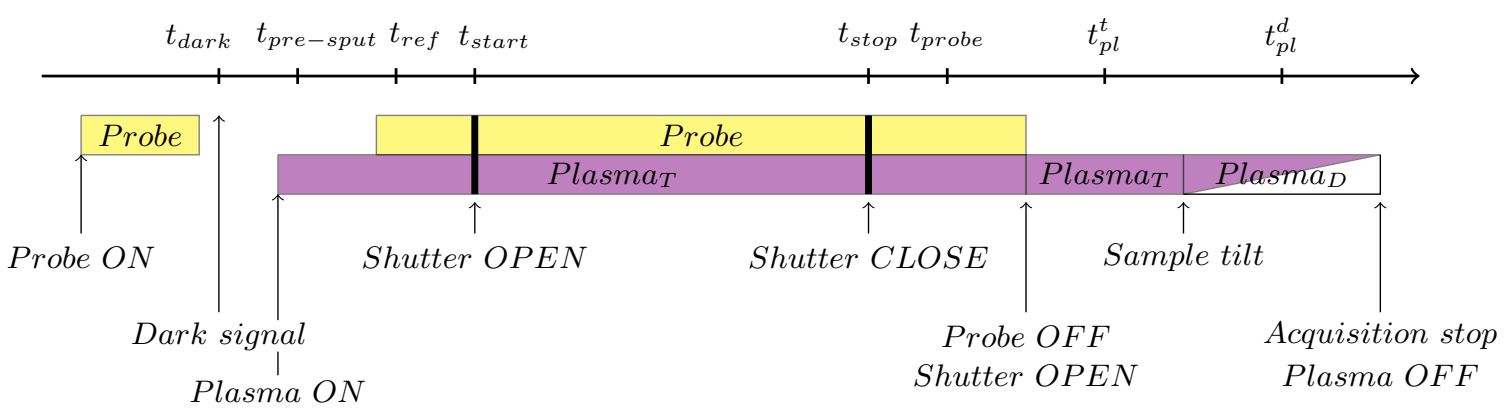

Figure 2. Timeline of the steps of plasma correction process. Yellow (violet, respectively) filling corresponds to times when the probe light (the plasma, respectively) is switched on.

the same experimental quantities as in the evaporation case will not result in pure differential reflectivity spectra. The raw experimental intensity $I(t)$ now contains the temporal contribution of the light emitted by the plasma $I_{p l}(t)$ which can be considered as a peculiar extra-component of dark signal:

$I(t)=\mathcal{R}(t) I_{\text {bench }}(t)+I_{\text {dark }}(t)+I_{p l}(t)$.

Assuming a stable optical bench $\left(I_{\text {bench }}(t) \simeq I_{\text {bench }}\right)$ and a stable actual dark signal without plasma $\left(I_{\text {dark }}(t) \simeq I\left(t_{\text {dark }}\right)=I_{\text {dark }}\right)$ and since there is no plasma signal before growth $\left(I_{p l}\left(t_{r e f}\right)=0\right)$,

$$
\begin{aligned}
\frac{\Delta I_{c}}{I_{c}}(t) & =\frac{I(t)-I\left(t_{\text {ref }}\right)}{I\left(t_{\text {ref }}\right)-I_{\text {dark }}} \\
& =\frac{\Delta \mathcal{R}}{\mathcal{R}}(t)+\frac{I_{p l}(t)}{\mathcal{R}\left(t_{\text {ref }}\right) I_{\text {bench }}} .
\end{aligned}
$$

In case of vapor deposition, the measured differential signal corrected from initial dark signal $\frac{\Delta I_{c}}{I_{c}}(t)$ equals to actual SDRS (equation 3). But in case of sputtering deposition, $\frac{\Delta I_{c}}{I_{c}}(t)$ contains a perturbation that depends on the detected intensity of plasma light with respect to the intensity of the probe source and also on the substrate reflectivity. In particular, plasma emission peaks even of low intensity can provide sizable contributions to this calculated ratio in the spectral range where the bare substrate reflectivity $\mathcal{R}\left(t_{\text {ref }}\right)$ is low. For example, this is likely to be the case with an incident angle close to the Brewster angle of the minimum of reflectivity in p-polarization. In practice, emission from the plasma can significantly alter the differential intensity spectrum. This is illustrated in figure 3 for a deposit of silver on a silicon wafer in the regime of discontinuous island growth. The distortion is especially relevant in the spectral region where the plasmonic response of the islands $[32,33,23,34,28,17]$ is observed. The dip at around $3.8 \mathrm{eV}$ in SDRS curve originates from the excitation of the normal dipole in the silver nanoparticles. In the presence of plasma, it is strongly impacted by two plasma peaks at 3.664 and $3.782 \mathrm{eV}$. As shown by equation 7 , the problem is minimized when $I_{p l}(t) \ll \mathcal{R}\left(t_{\text {ref }}\right) I_{\text {bench }}$, that is to say when a powerful probe lamp or a space filter are used. A naive strategy to remove $I_{p l}(t)$ would be to perform two deposits, with and without the probe light, and to subtract the two $\frac{\Delta I_{c}}{I_{c}}$ signals; but beyond the require- 


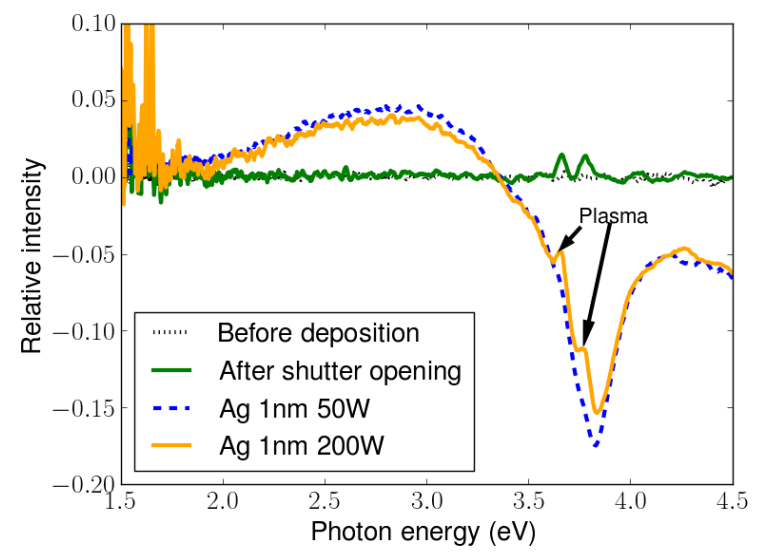

Figure 3. Differential signal $\frac{\Delta I_{c}}{I_{c}}(t)$ corrected from the initial dark after sputtering deposition of a $1 \mathrm{~nm}$ thick silver film on a silicon wafer. Measurements are made in p-polarization at an incident angle of $45^{\circ}$ for two deposition powers: 50 and $200 \mathrm{~W}$.

ment of performing two experiments, this procedure is very sensitive to reproducibility due to sample misalignment and growth conditions.

In order to define a single-deposition protocol and to get rid of the plasma contribution without loosing sensitivity, the light emitted by the plasma has been directly monitored over time showing its stability over the usual time scale of a deposit. In a second step, the spurious plasma signal $I_{p l}(t)$ collected by the optical bench in SDRS measurement conditions has been followed during deposition by switching off the probe light. Naturally, the absolute detected plasma spectrum (figure 4-a) depends on the geometry of the optical set-up but also on the deposition parameters, as for instance power (see figure 4-b), gas pressure or target-sample distance.

However, a systematic variation of intensity of some intrinsic peaks of emission from excited species has been observed during deposition. According to the literature [41, 42], the sharp and intense emission lines at $3.782 \mathrm{eV}(328 \mathrm{~nm}), 3.664 \mathrm{eV}(338 \mathrm{~nm}), 2.382 \mathrm{eV}$ $(521 \mathrm{~nm}), 2.275 \mathrm{eV}(545 \mathrm{~nm})$ are due to singly ionized $\mathrm{Ag}$ I and that at $2.956 \mathrm{eV}(419 \mathrm{~nm}), 1.652 \mathrm{eV}(750 \mathrm{~nm})$ and $1.551 \mathrm{eV}(800 \mathrm{~nm})$ are typical of Ar I. The intensity of some of those plasma-related features have been followed all along the growth process up to an average silver thickness of $100 \mathrm{~nm}$. The observed temporal variation is complex and strongly energy dependent (figure 5-a). But, in most of the thickness range, it turns out that this latter is an affine function of the reflectivity $\mathcal{R}_{f}(E)$ [43] expected for a continuous silver film on silicon at the emission peak energy (figure 5c). This latter was calculated in p-polarization at $45^{\circ}$ including the $2 \mathrm{~nm}$ thick native oxide on silicon using
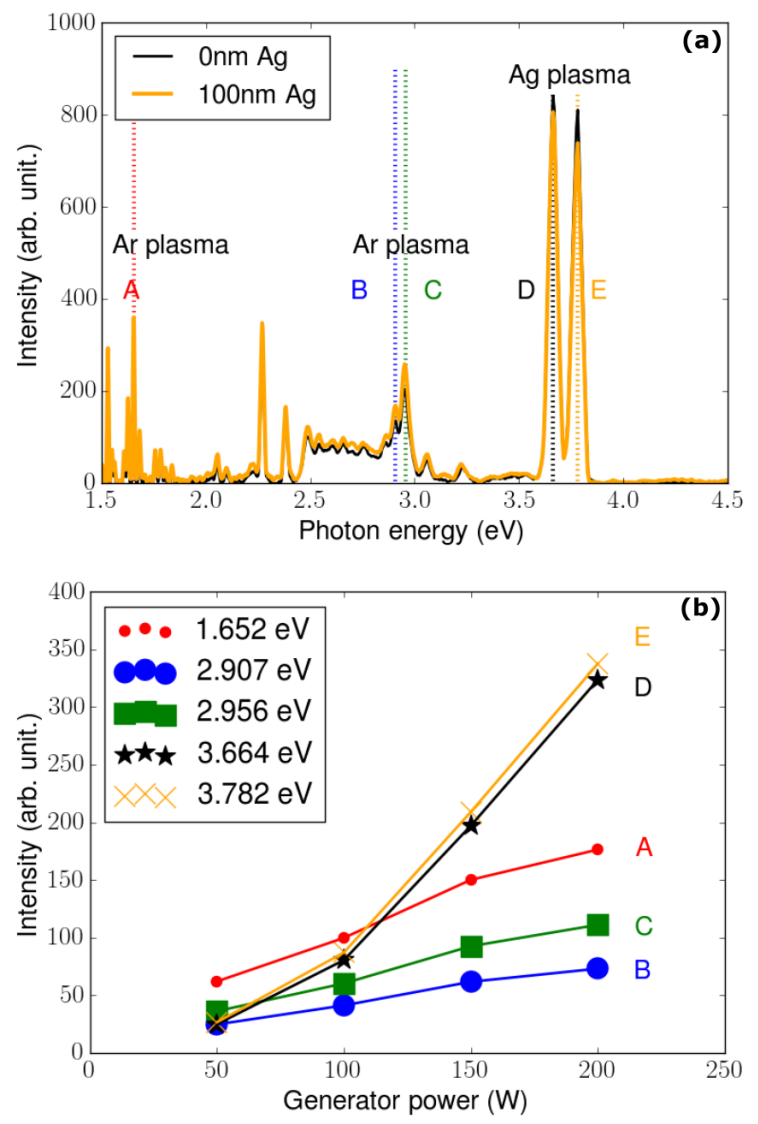

Figure 4. a) Raw spectra of plasma emission collected with the differential reflectivity set-up in absence of lamp illumination before and after deposition of a $100 \mathrm{~nm}$ thick layer on a silicon substrate (power $200 \mathrm{~W}$ ). b) Evolution of the integrated intensity of the indicated plasma peaks (A-E) as a function of generator power.

tabulated $\mathrm{Ag}, \mathrm{Si}$ and $\mathrm{SiO}_{2}$ indexes of refraction [44]. Below the percolation threshold (around 10-20 nm), the continuous morphology is questionable. But at higher thicknesses, the theoretical $\mathcal{R}_{f}(E)$ gives a fair estimate of the actual sample reflectivity. This experiment demonstrates clearly that the plasma contribution $I_{p l}(t)$ to the detected signal is the sum of two components. One reaches the detector directly $\left(I_{p l}^{d}\right)$ and stays constant over time while the other is reflected by the sample $\left(I_{p l}^{r} \mathcal{R}(t)\right)$ :

$I_{p l}^{t}(t)=I_{p l}^{d}+I_{p l}^{r} \mathcal{R}(t)$.

Although the plasma emission is isotropic, the contribution of mainly the reflection coefficient $\mathcal{R}(t)$ at the chamber incident angle is due (i) to the general overwhelming specular reflection over diffuse one on the growing layer and (ii) to the geometry of the light collection at the output of the optical bench. Due to the affine dependence of collected plasma light on sample reflectivity (equation 8), equation 7 can be 

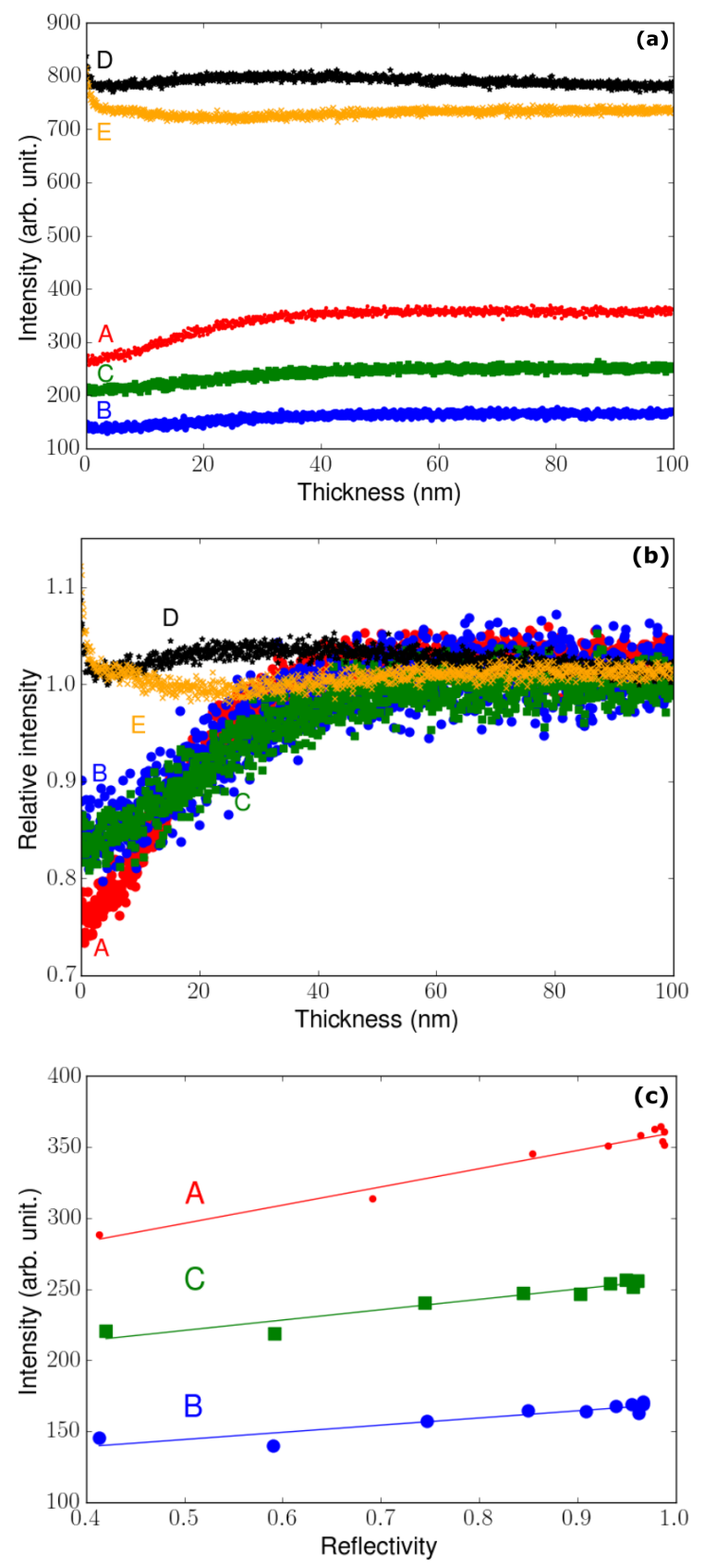

Figure 5. Evolution of intensities of plasma emission peaks during sputter deposition of $\mathrm{Ag}$ on $\mathrm{Si}$, without probe light, as a function of a) deposited thickness (or time) or b) once normalized to the end value. c) Same evolution but as a function of the theoretical reflectivity $\mathcal{R}_{f}(E)$ of a continuous silver film of similar thickness on silicon. In figure c, the dependence is nearly linear for all energies. Notice in figure-b the temporal stability of the plasma light emission in the regime of thick films. recasted into:

$$
\begin{aligned}
\frac{\Delta I_{c}}{I_{c}}(t) & =\frac{\Delta \mathcal{R}}{\mathcal{R}}(t)+\frac{I_{p l}^{d}+I_{p l}^{r} \mathcal{R}(t)}{\mathcal{R}\left(t_{\text {ref }}\right) I_{\text {bench }}} \\
& =\left(1+\frac{I_{p l}^{r}}{I_{\text {bench }}}\right) \frac{\Delta \mathcal{R}}{\mathcal{R}}(t)+\frac{I_{\text {pl }}\left(t_{\text {start }}^{+}\right)}{\mathcal{R}\left(t_{\text {ref }}\right) I_{\text {bench }}} \\
& =\alpha \frac{\Delta \mathcal{R}}{\mathcal{R}}(t)+\beta .
\end{aligned}
$$

Here $t=t_{\text {start }}^{+}$tags the time just after the beginning of growth when the plasma starts contributing to the dark signal (see figure 2). The two spectral factors $(\alpha, \beta)$ of equation 9 are constant with time and can be estimated through suitable measurements performed at the end of deposit. If the shutter in front of the cathode is closed but the sample remains illuminated by the probe lamp, equation 9 shows that:

$$
\frac{\Delta I_{c}}{I_{c}}\left(t_{\text {stop }}^{+}\right)=\frac{\Delta \mathcal{R}}{\mathcal{R}}\left(t_{\text {stop }}^{+}\right) \simeq \frac{\Delta \mathcal{R}}{\mathcal{R}}\left(t_{\text {stop }}^{-}\right),
$$

where the time $t_{\text {stop }}$ corresponds to the final thickness and \pm indicates the moment before $(-)$ and after $(+)$ the end of deposit (see figure 2). If the film reflectivity does not evolve after growth, the measurement at $t_{\text {stop }}^{+}$is equivalent to measurement at $t_{\text {probe }}$, when the reflected probe light is measured alone after deposition (see figure 2). Therefore if $\beta$ is known, equation 10 gives:

$\alpha=\frac{\frac{\Delta I_{c}}{I_{c}}\left(t_{\text {stop }}^{-}\right)-\beta}{\frac{\Delta \mathcal{R}}{\mathcal{R}}\left(t_{\text {stop }}^{-}\right)} \simeq \frac{\frac{\Delta I_{c}}{I_{c}}\left(t_{\text {stop }}^{-}\right)-\beta}{\frac{\Delta I_{c}}{I_{c}}\left(t_{\text {probe }}\right)}$.

In principle, $\beta$ can be directly obtained as the difference of the signal before $\left(t=t_{\text {ref }}\right)$ and after $\left(t=t_{\text {start }}^{+}\right)$ opening the shutter at the onset of film growth where the variation of sample reflectivity is negligible (see figure 2):

$\beta=\frac{I_{\text {pl }}\left(t_{\text {start }}^{+}\right)}{\mathcal{R}\left(t_{\text {ref }}\right) I_{\text {bench }}} \simeq \frac{I\left(t_{\text {start }}^{+}\right)-I\left(t_{\text {ref }}\right)}{I\left(t_{\text {ref }}\right)-I_{\text {dark }}}$.

However, it is difficult to define the time $t=t_{\text {start }}^{+}$. Measuring the initial plasma signal with a good statistics and grasping the first moments of deposition with a good sensitivity are antithetical. Therefore, based on the hypothesis of a stable plasma emission (see figure 5-b), it is better to use measurements performed at the end of deposit. The direct plasma contribution $I_{p l}^{d}$ can easily be determined by orienting the sample in such a way to get rid of the specular reflection in the detection direction (by a rotation and/or a translation for instance) and by switching on the sputtering plasma or reopening the deposition shutter (see figure $2, t=t_{p l}^{d}$ ). Moreover, since the relative variation of the plasma signal $I_{p l}(t)$ follows also the evolution of the sample reflectivity (see equation 8) i.e.:

$\frac{I_{p l}(t)-I_{p l}\left(t_{\text {start }}^{+}\right)}{I_{\text {pl }}\left(t_{\text {start }}^{+}\right)-I_{p l}^{d}}=\frac{\Delta \mathcal{R}}{\mathcal{R}}(t)$, 
one obtains at time $t=t_{\text {stop }}^{-}$just before the end of deposition:

$$
I_{p l}\left(t_{\text {start }}^{+}\right)=I_{p l}^{d}+\frac{I_{p l}\left(t_{\text {stop }}^{-}\right)-I_{p l}^{d}}{1+\frac{\Delta \mathcal{R}}{\mathcal{R}}\left(t_{\text {stop }}^{-}\right)} .
$$

By taking advantage of the nearly constant reflectivity of the layer in the regime of large thicknesses, the plasma contribution $I_{p l}\left(t_{s t o p}^{-}\right)$is nearly identical to that obtained after the end of the growth $I_{p l}^{t}$ at time $t=t_{p l}^{t}$ by switching off the probe light and restarting deposition. Actually, the closer $t_{p l}^{t}$ to $t_{s t o p}$ the better the approximation. Finally, introducing equation 14 in equation $12, \beta$ can be expressed through measurable quantities:

$$
\begin{aligned}
\beta & =\frac{I_{\text {pl }}\left(t_{\text {start }}^{+}\right)}{\mathcal{R}\left(t_{\text {ref }}\right) I_{\text {bench }}}=\frac{I_{\text {pl }}\left(t_{\text {start }}^{+}\right)}{I_{c}\left(t_{\text {ref }}\right)}= \\
& =\frac{I_{p l}^{d}}{I_{c}\left(t_{\text {ref }}\right)}+\frac{I_{p l}^{t}-I_{p l}^{d}}{\left[1+\frac{\Delta I_{c}}{I_{c}}\left(t_{\text {probe }}\right)\right] I_{c}\left(t_{\text {ref }}\right)} .
\end{aligned}
$$

Finally, the actual differential reflectivity signal is given by equation 11 :

$$
\frac{\Delta \mathcal{R}}{\mathcal{R}}(t)=\frac{\frac{\Delta I_{c}}{I_{c}}(t)-\beta}{\frac{\Delta I_{c}}{I_{c}}\left(t_{\text {stop }}^{-}\right)-\beta} \frac{\Delta I_{c}}{I_{c}}\left(t_{\text {probe }}\right) .
$$

with the above definition for $\beta$ (equation 15).

At last, one should be cautious about the subtracted dark signal involved in the definition of $I_{c}(t)$ depending on the status of the shutter in front of the cathode. If $t=t_{\text {dark }}$ is the time when no source of light is present (neither lamp nor plasma), the quantity $I\left(t_{\text {dark }}\right)$ is the actual dark signal that should be subtracted to all subsequent measurements (see figure 2). But, shutter closed, a constant parasitic light coming from the plasma emission reaches the detector. It is similar but not equal to the direct plasma contribution $I_{p l}^{d}$ during deposition because of the screening by the shutter. Calling $t=t_{\text {pre-sput }}$ the time when the plasma is ignited, the shutter closed but the lamp switched off before reference measurement $\left(t=t_{r e f}\right)$ and growth start $\left(t=t_{\text {start }}\right)$ (see figure 2), the definitions to be used are:

$$
\frac{\Delta I_{c}}{I_{c}}(t)=\frac{\left[I(t)-I\left(t_{\text {dark }}\right)\right]-\left[I\left(t_{\text {ref }}\right)-I\left(t_{\text {pre-sput }}\right)\right]}{I\left(t_{\text {ref }}\right)-I\left(t_{\text {pre-sput }}\right)},
$$

during deposition and:

$$
\frac{\Delta I_{c}}{I_{c}}(t)=\frac{I(t)-I\left(t_{r e f}\right)}{I\left(t_{r e f}\right)-I\left(t_{\text {pre-sput }}\right)},
$$

when the shutter is closed, in particular when $t=$ $t_{\text {probe }}$.

To sum up, the proposed measurement flow chart and the key quantities to disentangle the emission of plasma is shown in table 1 and the timeline in figure 2 .

\section{Growth of silver on silicon: a test case}

To illustrate the interest of the correction in conditions leading to a sizable plasma contribution (see figure 4), an experiment of silver deposition on a silicon substrate was performed at a power of $200 \mathrm{~W}$ corresponding to a fast deposition rate $\left(1.9 \mathrm{~nm} . \mathrm{s}^{-1}\right)$. Both $\mathrm{s}$ and $\mathrm{p}$ polarized signals were recorded with the previously described optical set-up following the protocol of measurement described in table 1 and in timeline figure 2 . The quantities of interest $I\left(t_{\text {dark }}\right), I\left(t_{\text {pre-sput }}\right), I\left(t_{\text {ref }}\right), \frac{\Delta I_{c}}{I_{c}}\left(t_{\text {probe }}\right), I_{p l}^{t}, I_{p l}^{d}$ for the plasma correction were obtained by averaging spectra over time frames respectively around $t_{\text {dark }}$, $t_{\text {pre-sput }}, t_{r e f}, t_{\text {probe }}, t_{p l}^{t}, t_{p l}^{d}$ to increase statistics. Actual dark correction was performed accordingly to equations 17-18. $\alpha$ and $\beta$ were obtained according to equations 11 and 15 in order to correct spectra following equations 9,16 .

Figure 6 compares corrected and uncorrected signals all along the growth process from island to percolated films. Spectra are dominated by (i) a low energy feature that shifts towards red and transforms into a continuous increase of reflectivity in the red part of the spectrum and by (ii) a high energy negative dip around $3.8 \mathrm{eV}$. They are assigned to the excitation of dipole-like plasmon resonances in growing nanoparticles, respectively parallel and perpendicular to the surface $[32,33,34,17,35]$. Since their splitting is indicative of the flattening of the particles, the evolution of the SDRS spectra is typical for nucleation, growth, coalescence and percolation of silver islands. The interest of the correction is particularly salient between 3.5 and $4 \mathrm{eV}$, in the region of the high energy plasmon resonance. Its position is particularly spoiled by the Ag plasma emissions $D, E$ (figure 4). The present methodology allows also to extract (i) the component of the plasma directly collected by the optical bench $I_{p l}^{d}$ as well as (ii) the total one $I_{p l}\left(t_{\text {start }}^{+}\right)$ including also the reflected part on the silicon wafer (figure 7 ) and (iii) the correction terms $\alpha, \beta$ (figure 8).

\section{Conclusion}

A methodology to correct UV-visible differential reflectivity spectra from spurious plasma emission during sputtering deposition has been proposed. Experiments without illumination light show that the collected plasma signal contains a direct component but also a reflected one that evolves with the deposit. A precise sequence of measurements in the regime of thick film, with or without probe light, plasma emission or sample allows to disentangle the plasma signal from that of interest through a numerical treatment. The protocol of correction is based on the assumption of a stable plasma emission over time. It also relies on a 

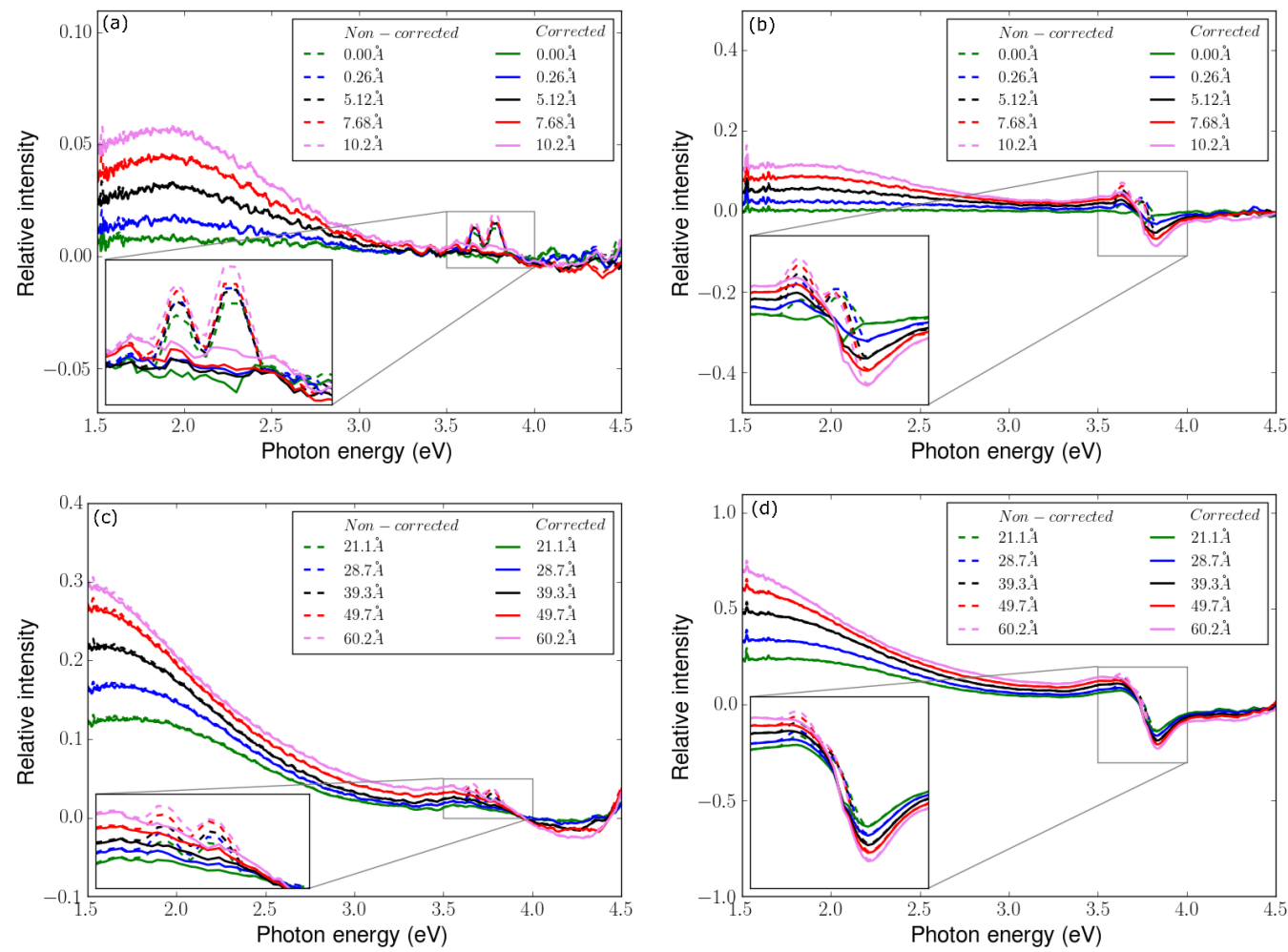

Figure 6. Comparison between raw $\Delta I_{c} / I_{c}$ and plasma-corrected $\Delta \mathcal{R} / \mathcal{R}$ signals along silver deposition on silicon (deposition power $200 \mathrm{~W}$ ). Left (right, respectively) column: s-polarization (p-polarization, respectively) at a)b) sub-nanometer and c)d) and nanometer-scale average thicknesses.

reference measurement in the thick film regime where the reflectivity does not change dramatically with thickness. In this respect, absorbing layers like metals are more suited that transparent dielectrics because of potential interference fringes. Nevertheless, by providing high quality data, this proposed correction and the fast acquisition of modern spectrometers pave the way to a better understanding of the first stages of thin film growth by optical spectroscopy in the harsh conditions of sputtering deposition.

\section{References}

[1] Fukuda K, Lim SHM, Anders A. 2008 Coalescence of magnetron-sputtered silver islands affected by transition metal seeding $(\mathrm{Ni}, \mathrm{Cr}, \mathrm{Nb}, \mathrm{Zr}, \mathrm{Mo}, \mathrm{W}, \mathrm{Ta})$ and other parameters. Thin Solid Films 516 4546-52

[2] Abadias G, Simonot L, Colin JJ, Michel A, Camelio S, Babonneau D. 2015 Volmer-Weber growth stages of polycrystalline metal films probed by in situ and realtime optical diagnostics. Appl. Phys. Lett. 107183105

[3] Chason E, Guduru PR. 2016 Tutorial: understanding residual stress in polycristalline thin films through realtime measurements and physical models. J. Appl. Phys. 119191101

[4] Dietz N. 2001 Real-time optical characterization of thin film growth. Mat. Sci. Eng. B 87 1-22

[5] Yamamoto M, Namioka T. 1992 In situ ellipsometric study of optical properties of ultrathin films. Appl. Opt. 31 $1612-21$

[6] Nguyen HV, An I, Collins RW. 1992 Evolution of the optical functions of aluminium films during nucleation and growth determined by real-time spectroscopic ellipsometry. Phys. Rev. Lett. 68 994-7

[7] Zayer NK, Greef R, Roger K, Grellier AJC, Pannell CN. 1999 In situ monitoring of sputtered zinc oxide films for piezoelectric transducers. Thin Solid Films 352 179-84

[8] Dalacu D, Martinu L. 2000 Spectroellipsometric characterization of plasma-deposited $\mathrm{Au} / \mathrm{SiO}_{2}$ nanocomposite films. J. Appl. Phys. 87 228-35

[9] Armelao L, Barreca D, Bottaro G, Gasparotto A, Losurdo M, Tondello E. 2005 RF-sputtering of gold silica surfaces: evolution from clusters to continuosu films. Materials Science and Engineering C 25 599-603

[10] Oates TWH, Mücklich A. 2005 Evolution of plasmon resonances during plasma deposition of silver nanoparticles. Nanotechnology. 16 2606-11

[11] Oates T, Christalle E. 2007 Real-time spectroscopy ellipsometry of silver nanoparticle formation in poly(vinyl alcohol) thin films. J. Phys. Chem. C 111 182-7

[12] Beyene HT, Weber JW, Verheijen MA, van de Sanden MCM, Creatore M. 2012 Real time in situ spectroscopic ellipsometry of the growth and plasmonic properties of $\mathrm{Au}$ nanoparticles on $\mathrm{SiO}_{2}$. Nano Res. 5 513-20

[13] Simonot L, Babonneau D, Camelio S, Lantiat D, Gurin P, Lamongie B, et al. 2010 In situ optical spectroscopy during deposition of $\mathrm{Ag}: \mathrm{Si}_{3} \mathrm{~N}_{4}$ nanocomposite films by magnetron sputtering. Thin Solid Films 518 2637-43

[14] Bulír J, Novotný M, Lynnykova A, Lančcok J. 2011 Preparation of nanostructured ultrathin silver layer. J. 

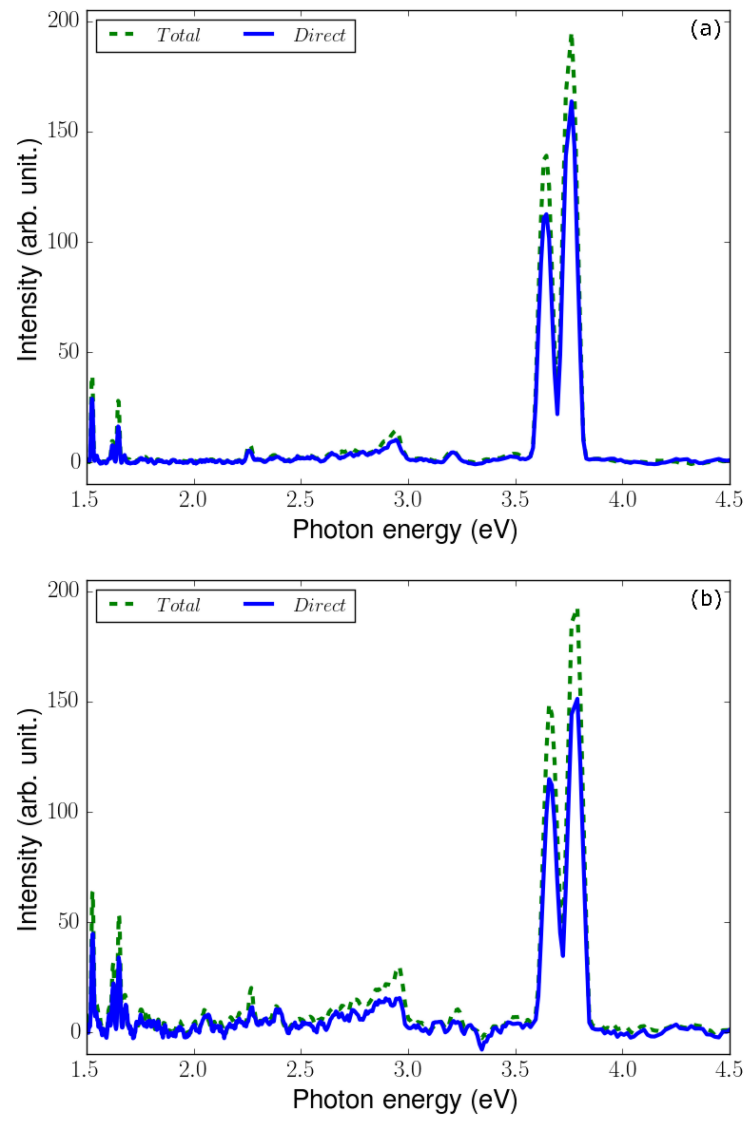

Figure 7. Direct $I_{p l}^{d}$ and total $I_{p l}^{t}$ components of the plasma emission recorded on a) s and b) p channels of the SDRS set-up.

Nanophotonics 5051511

[15] Antad V, Simonot L, Babonneau D. 2013 Tuning the surface plasmon resonance of silver nanoclusters by oxygen exposure and low-energy plasma annealing. Nanotechnology 24045606

[16] Antad V, Simonot L, Babonneau D. 2014 Influence of low-energy plasma annealing on structural and optical properties of silver nanoclusters grown by magnetron sputtering. J. Nanopart. Res. 162328

[17] Grachev S, De Grazia M, Barthel E, Søndergård E, Lazzari R. 2013 Real time monitoring of nanoparticle film growth at high deposition rate with optical spectroscopy of plasmon resonances. J. Phys. D. Appl. Phys. 46 375305-15

[18] Meng F, Pucci A. 2007 Growth of silver on $\mathrm{MgO}(001)$ and infrared optical properties. Phys. status solidi 244 373949

[19] Barreca D, Gasparotto A, Maragno C, Tondello E, Gialanella S. 2005 Silica-supported silver nanoparticles: tailoring of structure-property relationships. J. Appl. Phys. 97 054311-17

[20] McIntyre JDE, Aspnes DE. 1971 Differential reflection spectroscopy of very thin surface films. Surf. Sci. 24 417-34

[21] Bagchi A, Barrera RG, Fuchs R. 1982 Local-field effect in optical reflectance from adsorbed overlayers. Phys. Rev. B. 25 7086-96.

[22] Bedeaux D, Vlieger J. 2001 Optical Properties of Surfaces. London: Imperial College Press
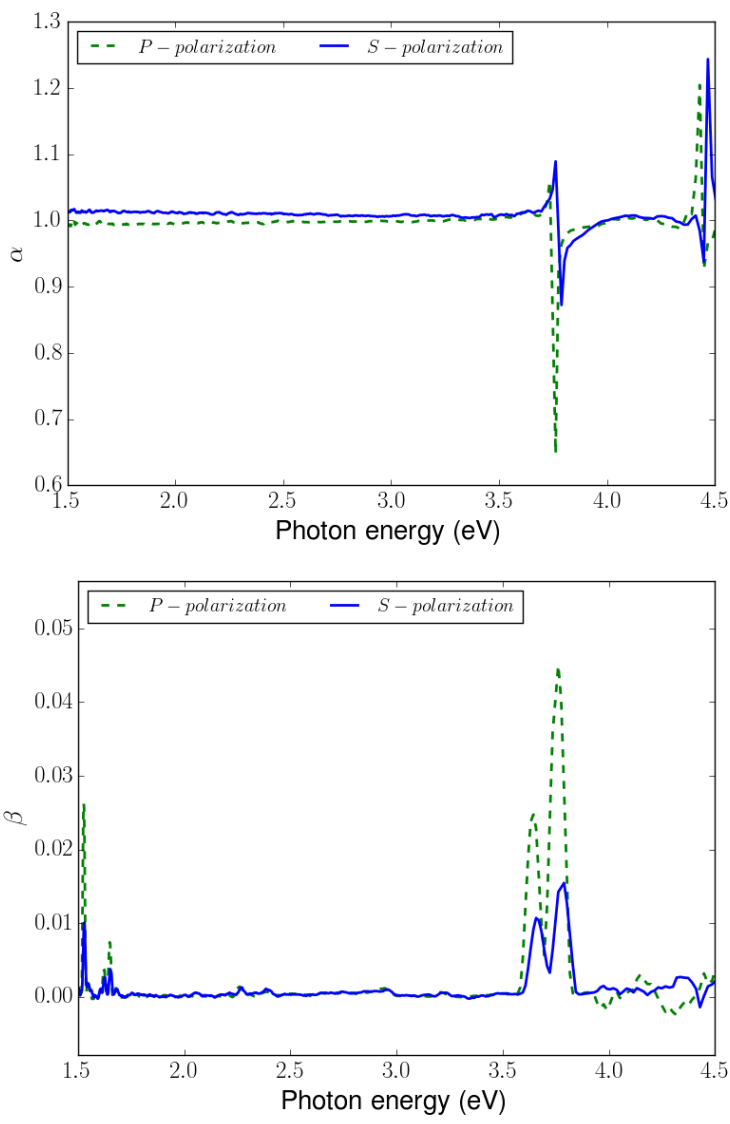

Figure 8. $\alpha, \beta$ correction terms obtained after data treatment (see text for definitions).

[23] Lazzari R, Jupille J. 2011 Quantitative analysis of nanoparticle growth through plasmonics. Nanotechnology 22 445703

[24] Seki H, Itoh U. 1980 Oscillating beam spectrometer. Rev. Sci. Instrum. $5122-6$

[25] Hummel RE. 1983 Differential reflectometry and its application to the study of alloys, ordering, corrosion, and surface properties. Phys. Status Solidi 76 11-44

[26] Pollak FH, Shen H. 1993 Modulation spectroscopy of semiconductors: bulk/thin film, microstructures, surfaces/interfaces and devices. Mater. Sci. Eng. R. 10 275-374

[27] Borensztein Y, Abelès F. 1985 Surface reflectance spectroscopy: its application to the study of very thin films. Thin Solid Films 125 129-142

[28] Lazzari R, Simonsen I, Jupille J. 2014 Interfacial susceptibilities in nanoplasmonics via inversion of Fresnel coefficients. Plasmonics 9 261-272

[29] Navarro-Quezada A, Aiglinger M, Ghanbari E, Wagner T, Zeppenfeld P. 2015 Polarization-dependent differential reflectance spectroscopy for real-time monitoring of organic thin film growth. Rev. Sci. Instrum. 86113108

[30] Proehl H, Nitsche R, Dienel T, Leo K, Fritz T. 2005 In situ differential reflectance spectroscopy of thin crystalline films of PTCDA on different substrates. Phys. Rev. B 71165207

[31] Forker R, Gruenewald M, Fritz T. 2012 Optical differential reflectance spectroscopy on thin molecular films. Annu. Reports Sec. "C" (Physical Chem.) 108 34-68 
[32] Lazzari R, Jupille J. 2005 Interfacial chemistry and wetting of metallic films on the hydroxylated $\alpha-\mathrm{Al}_{2} \mathrm{O}_{3}(0001)$ surface. Phys. Rev. B 71045409

[33] Lazzari R, Renaud G, Revenant C, Jupille J, Borenstzein Y. 2009 Adhesion of growing nanoparticles at a glance: Surface differential reflectivity spectroscopy and grazing incidence small angle X-ray scattering. Phys. Rev. B. 79 125428

[34] Lazzari R, Jupille J. 2012 Growth kinetics and sizedependent wetting of $\mathrm{Ag} / \alpha-\mathrm{Al}_{2} \mathrm{O}_{3}(0001)$ nanoparticles studied via the plasmonic response. Nanotechnology 23 135707

[35] Lazzari R, Goniakowski J, Cabailh G, Cavallotti R, Trcera N, Jupille J, et al. 2016 Surface and epitaxial stress for supported metal clusters. Nano Lett. 16 2574-79

[36] Simonsen I, Lazzari R, Jupille J, Roux S. 2000 Numerical modelling of the optical response of supported metallic particles. Phys. Rev. B 61 7722-33

[37] Lazzari R, Simonsen I, Bedeaux D, Vlieger J, Jupille J. 2001 Polarizability of truncated spheroidal island supported by a substrate : models and applications. Eur. Phys. J. B 24 267-84

[38] Lazzari R, Simonsen I. 2002 GranFilm : a software for calculating thin-layer dielectric properties and Fresnel coefficients. Thin Solid Films 419 124-36

[39] Lazzari R, Jupille J, Cavallotti R, Simonsen I. 2014 Modelfree unraveling of supported nanoparticles plasmon resonance modes. J. Phys. Chem C. 118 7032-48

[40] Sell DD. A sensitive spectrophotometer for optical reflectance and transmittance measurements. Review of Scientific Instruments. 1970;9(8):1926-1930.

[41] Wagatsuma K, Hirokawa K. 1985 Characterization of atomic emission lines from argon, neon, and nitrogen glow discharge. Anal. Chem. 57 2901-07

[42] Atomic spectra database; https://www.nist.gov/pml/atomic-spectra-database.

[43] Born M, Wolf E. 1999 Principles of Optics. vol. 7 th ed. (Cambridge University Press)

[44] Palik ED. 1985 Handbook of Optical Constants of Solids. Academic Press 